\title{
Dual oxidase 1: A predictive tool for the prognosis of hepatocellular carcinoma patients
}

\author{
SHENGSEN CHEN, QINGXIA LING, KANGKANG YU, CHONG HUANG, NING LI, JIANMING ZHENG, \\ SUXIA BAO, QI CHENG, MENGQI ZHU and MINGQUAN CHEN
}

Department of Infectious Diseases and Hepatology, Huashan Hospital, Fudan University, Shanghai 200040, P.R. China

Received December 17, 2015; Accepted February 1, 2016

DOI: $10.3892 /$ or.2016.4745

\begin{abstract}
Dual oxidase 1 (DUOX1), which is the main source of reactive oxygen species (ROS) production in the airway, can be silenced in human lung cancer and hepatocellular carcinomas. However, the prognostic value of DUOX1 expression in hepatocellular carcinoma patients is still unclear. We investigated the prognostic value of DUOX1 expression in liver cancer patients. DUOX1 mRNA expression was determined in tumor tissues and non-tumor tissues by real-time PCR. For evaluation of the prognostic value of DUOX1 expression, Kaplan-Meier method and Cox's proportional hazards model (univariate analysis and multivariate analysis) were employed. A simple risk score was devised by using significant variables obtained from the Cox's regression analysis to further predict the HCC patient prognosis. We observed a reduced DUOX1 mRNA level in the cancer tissues in comparison to the non-cancer tissues. More importantly, Kaplan-Meier analysis showed that patients with high DUOX1 expression had longer disease-free survival and overall survival compared with those with low expression of DUOX1. Cox's regression analysis indicated that DUOX1 expression, age, and intrahepatic metastasis may be significant prognostic factors for disease-free survival and overall survival. Finally, we found that patients with total scores of $>2$ and $>1$ were more likely to relapse and succumb to the disease than patients whose total scores were $\leq 2$ and $\leq 1$. In conclusion, DUOX1 expression in liver tumors is a potential prognostic tool for patients. The risk scoring system is useful for predicting the survival of liver cancer patients after tumor resection.
\end{abstract}

\section{Introduction}

Hepatocellular carcinoma (HCC) is one of the most common cancers worldwide, especially in Asia, with a high mortality rate (1). It is challenging to evaluate the prognosis of HCC

Correspondence to: Dr Mingquan Chen, Department of Infectious Diseases and Hepatology, Huashan Hospital, Fudan University, 12 Middle Urumqi Road, Shanghai 200040, P.R. China

E-mail: bagews@163.com

Key words: dual oxidase 1, hepatocellular carcinoma, biomarker, risk scoring system, prognosis patients. Based on molecular profiling, several prognostic markers for HCC are used in the clinic (2), but only a few genes have been identified as useful.

Dual oxidase 1 (DUOX1) is a key phenotype of the NADPH-oxidase (NOX) family, and the main function of this gene is reactive oxygen species (ROS) production (3). DUOX1 is predominantly found in the thyroid, where it is involved in the synthesis of thyroid hormones (4). It is also highly expressed in normal epithelial cells in the airway, pancreas, placenta, prostate, testis and salivary gland $(3,5)$. Recent research indicates that DUOX1 may function as a selective tumor-suppressor gene (TSG) during tumor initiation and progression. In lung cancer cells, DUOX1 is frequently silenced by its promoter hypermethylation (6). In poorly differentiated follicular thyroid carcinoma, a high expression of DUOX1 is associated with a reduced risk of death (7). Moreover, our previous study (8) found that DUOX1 expression is also frequently decreased in most liver cancer cell lines and primary HCC tissues compared to its expression in non-tumor tissues, and silencing of DUOX1 gene expression is mediated by promoter hypermethylation.

Reactive oxygen species (ROS), chemically reactive molecules containing oxygen including oxygen ions and peroxides, are the key mediators of cellular oxidative stress and redox dysregulation involved in cancer initiation and progression (9). For a long time, ROS were considered oncogenic since they were implicated in cancer progression and metastasis (9). Increased ROS levels contribute to genetic instability and cancer initiation and progression $(10,11)$. Thus, it is widely accepted that constitutively elevated levels of cellular oxidative stress and dependence on mitogenic and anti-apoptotic ROS signaling in cancer cells are involved in carcinogenesis (12). Paradoxically, apart from being involved in proliferative, anti-apoptotic, metastatic and angiogenic signaling, ROS may also exert cytotoxic and pro-apoptotic functions that would limit tumorigenicity and malignant progression $(13,14)$. We previously reported that DUOX1 acts as a TSG to suppress tumor cell growth through the induction of $\mathrm{G} 2 / \mathrm{M}$ phase cell cycle arrest and an increase in ROS generation (8). However, our previous research has not identified associations between clinical outcomes and DUOX1 expression.

We hypothesized that DUOX1 could be used as a pathological and prognostic biomarker for HCC patients. Therefore, we investigated the expression of DUOX1 in a set of HCC 
specimens. The results validated the relevance of DUOX1 expression to HCC clinical outcomes.

\section{Materials and methods}

Patient cohort and specimens. Seventy-two patients (56 males and 16 females) from Huashan Hospital (Shanghai, China) were included in this study. All the patients underwent radical hepatic resection for HCC between 2008 and 2010. The age of the patients ranged from 16 to 84 years [mean \pm standard deviation (SD), 53.67 \pm 12.30 years]. The criteria for radicality have been published (15). None of the patients in this study received any preoperative chemotherapy or embolization therapy. The tumor tissues and the adjacent non-tumor tissues were collected from these patients above as frozen samples. The distance between adjacent non-tumor tissue and tumor tissue boundary was $2 \mathrm{~cm}$, beyond which was regarded as distant normal tissue. The selected tumor areas consisted of more than $80 \%$ tumor cells which was confirmed by histologic examination. Classification of the tumor stage using the Tumor-Node-Metastasis (TNM) stage was based on the 7th edition of the American Joint Committee on Cancer (AJCC) Cancer Staging Manual (16).

Ethic statements. All patients were given informed consent for obtaining the study specimens. Experiments and procedures were in accordance with the Helsinki Declaration of 1975, and approved by the Human Ethics Committee of Shanghai Fudan University.

Follow-up. Follow-up ended at death or June 1st, 2013, whichever came first. Follow-up imaging was performed every 3-6 months for 2 years and then every 6-12 months. According to the revised Response Evaluation Criteria in Solid Tumors (RECIST) guidelines (version 1.1) (17), the appearance of one or more new malignant lesions on multiphase computed tomography (CT) scan or magnetic resonance (MR) imaging denoted disease progression. Disease-free survival (DFS) was defined as the time period from the date of surgical operation to the first cancer recurrence (local or distant). Overall survival (OAS) was calculated from the date of cancer resection to death or the last contact.

RNA/DNA extraction and reverse transcription. Total RNA and genomic DNA from the human tissue samples were extracted using TRIzol reagent (Invitrogen) according to the manufacturer's instructions, and their concentrations were quantified by NanoDrop 1000 (NanoDrop, Wilmington, DE, USA). A reverse transcription reaction was performed using $1 \mu \mathrm{g}$ of total RNA with the High Capacity cDNA Reverse Transcription kit (SYBR qPCR RT Mix, FSQ-101; Toyobo).

Quantitative real-time PCR. The mRNA levels of DUOX1 were determined by real-time PCR using SYBR Green Master Mix kit and ABI 7500 Real-Time PCR system (Applied Biosystems, Foster City, CA, USA). Glyceraldehyde-3-phosphate dehydrogenase (GAPDH) was used as an internal control of RNA integrity. The $2^{-\Delta \Delta c t}$ method was used to analyze the relative changes in DUOX1 expression from the real-time PCR experiments (18). Real-time PCR was performed in triplicate.
Primers used for DUOX1 were: DUOX1 forward, 5'-CCACCA GGAGTGGCATAAGT-3' and reverse, 5'-CAGCTGACGGAT GACTTGAA-3' (110-bp product).

Statistical analysis. Kruskal-Wallis test was used to examine the statistical differences among three groups or more. The Mann-Whitney U-test was used to compare continuous variables between two groups. The diagnostic performance of DUOX1 was assessed by receiver operating characteristic (ROC) curve and the area under the ROC curve (AUC). Survival curves were plotted using the Kaplan-Meier method, and the statistical significance between groups was determined by taking the log-rank test. Independent variables predicting survival were evaluated by conducting a multiple stepwise regression analysis with the Cox model. A simple risk score was devised by using significant variables obtained from the multiple stepwise Cox's regression analysis with $\mathrm{P}<0.05$. The discrimination capabilities of the simple risk score were also presented by ROC curve and AUC. The optimal cut-off value was determined to maximize the sum of the sensitivity and specificity. All statistical tests were two-sided, and p-values $<0.05$ were considered as statistically significant. The statistical analyses were performed using SPSS version 21.0, MedCalc version 11.4 and GraphPad Prism version 5.0.

\section{Results}

Correlation of DUOX1 expression and the clinicopathological features. We enrolled 72 HCC patients in this study. The median age of the liver cancer patients was 53.67 years (range, 16-84 years). The HCC patients were grouped according to tissue type (tumor tissue vs. non-tumor tissue), HBsAg expression (positive vs. negative), histological grade (grade 1, 2 and 3 groups), tumor stage (stage I, II, III and IV groups) and hepatic cirrhosis (yes vs. no), respectively. We confirmed the difference in DUOX1 expression in these groups (Fig. 1). DUOX1 mRNA expression was significantly downregulated in the human primary HCC tissues when compared with that in the adjacent non-tumor tissues (Fig. 1A). The expression of DUOX1 mRNA was also significantly related to HBsAg expression ( $p=0.009$, Fig. 1B) and hepatic cirrhosis ( $p=0.018$, Fig. 1E). Histological grade and tumor stage were not correlated with DUOX1 mRNA levels (Fig. 1C and D).

Diagnostic performance of DUOXI and determination of the optimal cut-off value of DUOX1 mRNA levels. We considered death (yes vs. no) and recurrence (yes vs. no) as final diagnosis separately; DUOX1 expression was regarded as a diagnostic test. Then two ROC curves were plotted by software MedCalc11.4 to evaluate the predictive efficacy of DUOX1 for HCC patient survival (Fig. 2). The optimal cut-off values for DUOX1 expression were 3.128 and 3.468 according to the ROC curve for recurrence (Fig. 2A) and for death (Fig. 2B), respectively. Corresponding diagnostic indices were as follows: sensitivity 77.14 and $77.78 \%$, specificity 54.05 and $62.96 \%$, AUC 0.653 and 0.749 . Based on the optimal cut-off values for DUOX1 expression, patients were further categorized into two groups to evaluate DFS ( $\geq 3.128$, high expression vs. $<3.128$, low expression) and OAS ( $\geq 3.468$, high expression vs. $<3.468$, low expression). 

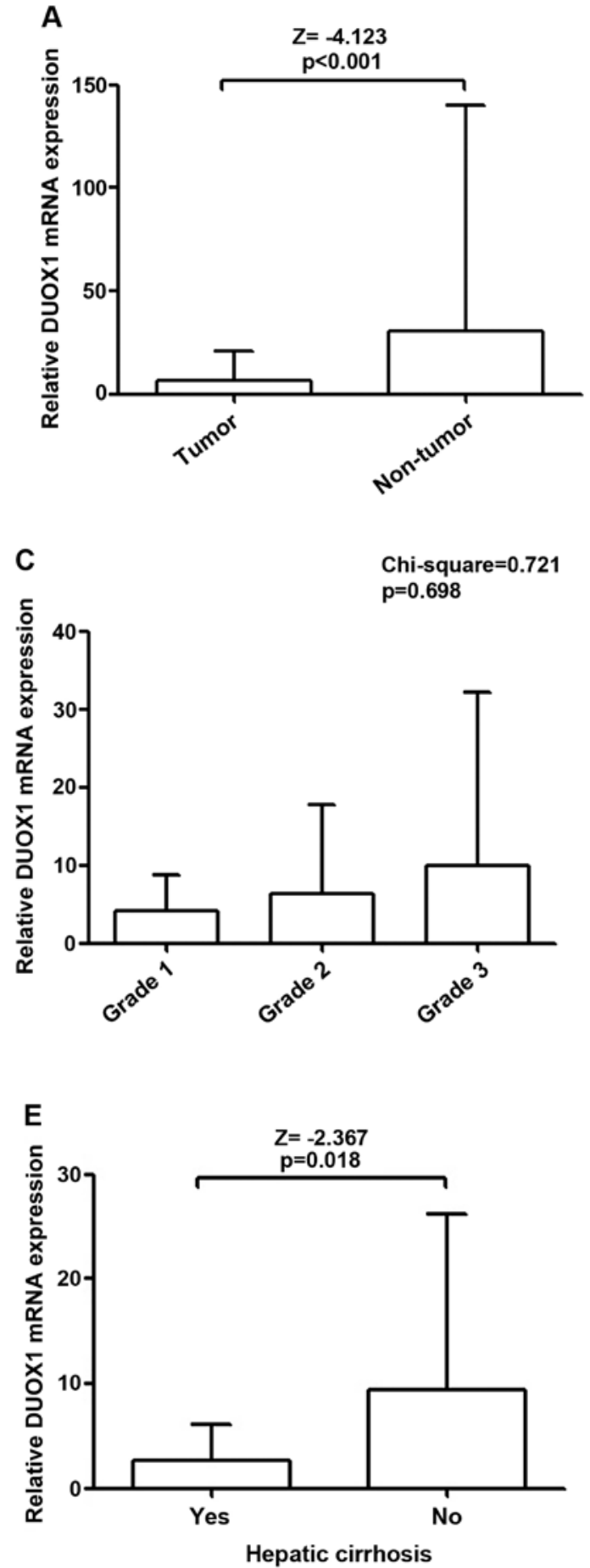

Relationship between disease-free survival, overall survival and clinicopathological factors in the HCC patients. Subsequently, we used the Kaplan-Meier method to further investigate the impact of clinical factors on DFS and OAS. As shown in Figs. 3A and 4A, patients with high DUOX1 expression tended to have prolonged DFS and OAS compared with those with low DUOX1 expression. Figs. 3B and $F$ and $4 \mathrm{~B}$ and $\mathrm{F}$ show that HBsAg expression and age [60 years was taken as the cut-off value according to Gokcan's et al (19)] were significantly correlated with DFS and OAS. However, it is clear from Figs. 3D and E and 4D and E that intrahepatic metastasis was correlated with OAS only and TNM stage
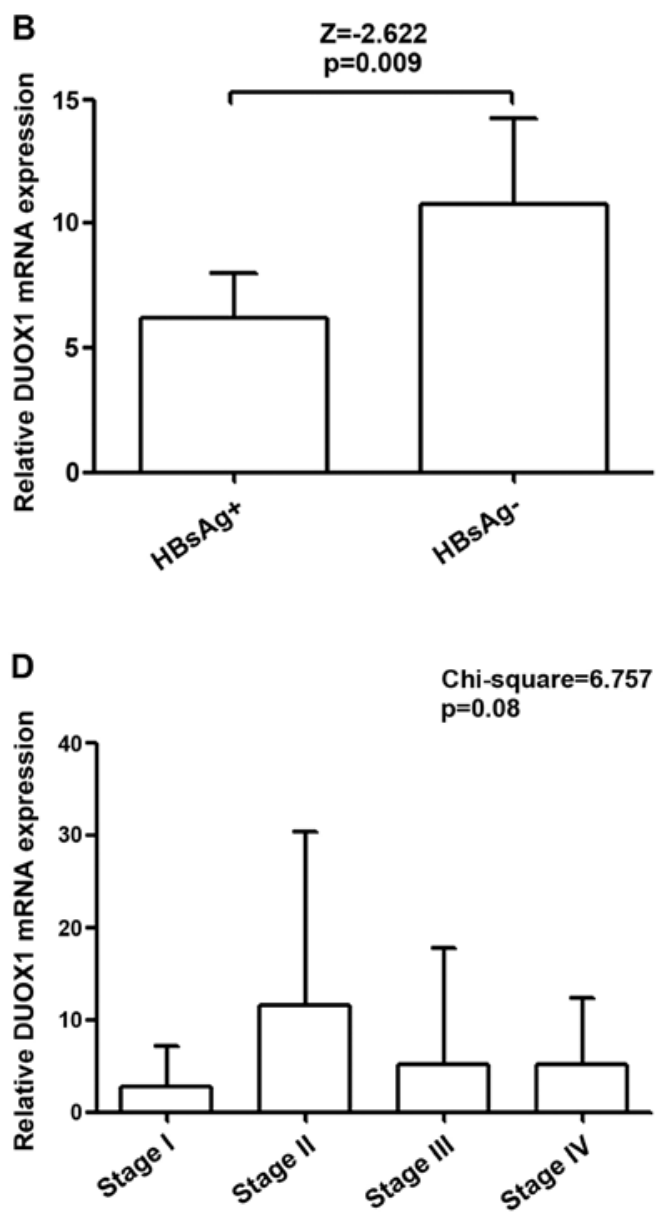

Figure 1. Clinicopathologic features and expression of DUOX1. The expression of DUOX1 in HCC tissues and adjacent non-tumor tissues was determined by real-time PCR. (A) Seventy-two pairs of samples were obtained from liver tissues, including tumor tissues and adjacent non-tumor tissues; p-value according to the Mann-Whitney U-test. (B) The expression of DUOX1 mRNA in $\mathrm{HBsAg}^{+}$and $\mathrm{HBsAg}^{-}$groups (p-value according to the Mann-Whitney U-test). (C) The expression of DUOX1 mRNA in different histological grades (three-tier grading scheme) of primary HCC tissues (p-value according to the Kruskal-Wallis test). (D) The expression of DUOX1 mRNA in different TNM stages of primary HCC tissues (p-value according to the Kruskal-Wallis test). (E) The expression of DUOX1 mRNA in hepatic cirrhosis and non-hepatic cirrhosis groups (p-value according to the Mann-Whitney U-test). seemed merely related to DFS. Finally, histological grade had no correlation with DFS (Fig. 3C) and OAS (Fig. 4C).

Univariate analysis and multivariate analysis with Cox proportional hazards model. Furthermore, the univariate Cox's proportional hazards model, in which the clinical factors such as gender, HBsAg, tumor size [5 $\mathrm{cm}$ was considered as cut-off value according to Hwang's et al (20)], age, intrahepatic metastasis, histological grade, tumor stage, and DUOX1 expression were respectively included, showed that low expression of DUOX1 was an independent prognostic factor for DFS $(R R=3.65, p=0.001)$ and OAS $(R R=3.69, p<0.001)$ 

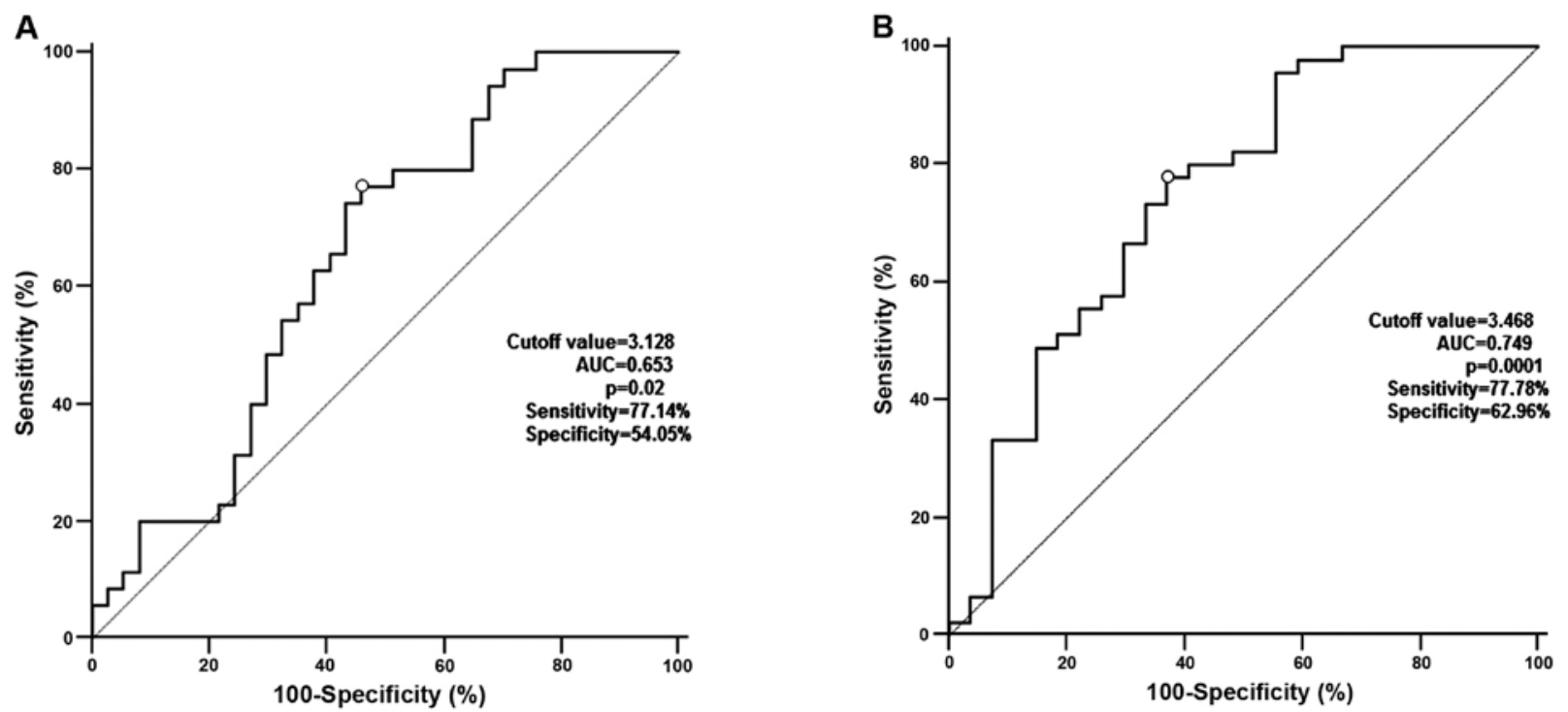

Figure 2. ROC curves of DUOX1 expression to indentify the cut-off value of the relative DUOX1 mRNA level. (A) ROC curve of DUOX1 expression for disease-free survival. (B) ROC curve of DUOX1 expression for overall survival.

in hepatic carcinoma patients (Table I). Notably, tumor stage and HBsAg expression were merely correlated with DFS ( $R R=2.10, p=0.030$, and $R R=8.82, p=0.032$ ), and showed no association with OAS. The results also showed that age was an independent unfavorable prognostic factor for DFS and OAS (Table I).

The multivariable analysis including the significant prognostic factors in the univariate analysis for DFS and OAS after radical resection for $\mathrm{HCC}$ is summarized in Tables II and III. The expression of DUOX1 was found to be one of the independent risk factors in the multivariable analysis for DFS ( $\mathrm{p}=0.002, \mathrm{RR}=3.67$; Table II) and OAS $(p<0.001, R R=4.63$; Table III). Age and intrahepatic metastasis were also significantly correlated with DFS (Table II) and OAS (Table III), while elderly age appeared to have a more significant impact on DFS ( $\geq 60$ years vs. $<60$ years, $R R=3.86$, $\mathrm{p}=0.007)$ and OAS ( $\geq 60$ years vs. $<60$ years, $R R=4.55$, $\mathrm{p}=0.001)$.

A simple risk score for predicting HCC patient prognosis. A simple risk score was then devised by using significant variables in the Cox model with $\mathrm{P}<0.05$. The score is the weighted sum of those variables for which the weights were defined as the quotient (rounded to the nearest integer) of the corresponding estimated coefficients from a Cox's regression analysis divided by the smallest regression coefficient in the same Cox model (Tables IV and V). The total score ranging from 0 to 4 was used to predict DFS. OAS was predicted by the total score ranging from 0 to 3 . HCC patients were divided into two groups by the endpoint of DFS (recurrence: yes or no) or endpoint of OAS (death: yes or no), and the total score was considered as a diagnostic test. Then two ROC curves were performed by software MedCalc11.4 (Fig. 5). The optimal cut-off points of the two ROC curves were score 1 (OAS prediction score, Fig. 5B) and score 2 (DFS prediction score, Fig. 5A) separately. For clinical and informative application, the patients were further categorized into two risk groups to evaluate DFS (total score $\leq 2$ vs. total score $>2$ ) and OAS (total score $\leq 1$ vs. total score $>1$ ). From Fig. 6, it was determined that patients whose total score was more than 2 were more likely to relapse and patients with a total score more than 1 were apt to succumb to the disease than patients whose score was less than 2 and 1. By applying the cut-off point of the two ROC curves, the sensitivity and specificity to predict the death of liver cancer patients after surgery were 73.33 and $77.78 \%$, and to predict the recurrence of HCC patients after operation were 62.86 and $72.97 \%$. The AUC of the ROC curve for DFS was 0.706 (Fig. 5A) and for OAS was 0.798 (Fig. 5B).

\section{Discussion}

Generally, the increase in ROS production in epithelial cells is mainly attributed to mitochondrial superoxide production (21). However, coordinated expression of DUOX1 and its maturation factor DUOXA1 in some epithelial cancer cells suggests that the intracellular level of ROS (superoxide and hydrogen peroxide) in epithelial cells may be partially controlled by the dual oxidases (5). Regardless of the role of ROS in cancer initiation and progression, a recent report linked intracellular ROS accumulation to the establishment of senescence, connecting ROS to tumor suppression (22). This is in contrast to the well-described tumor-promoting ROS activities which have been implicated in enhanced cell proliferation and metastasis. Moreover, it has been demonstrated that cisplatin apoptogenicity depends on the formation of ROS and occurs independently of nuclear DNA damage, suggesting that apoptogenic oxidative stress is the crucial mechanism of cisplatin-induced cancer cell death (23). Moreover, our previous study also demonstrated that DUOX1 exerted cytotoxic and pro-apoptotic functions and suppressed tumorigenicity and malignant progression by enhancing the production of intracellular ROS (8).

It has been well established that cell cycle checkpoints are important control mechanisms in maintaining tissue homeo- 
A

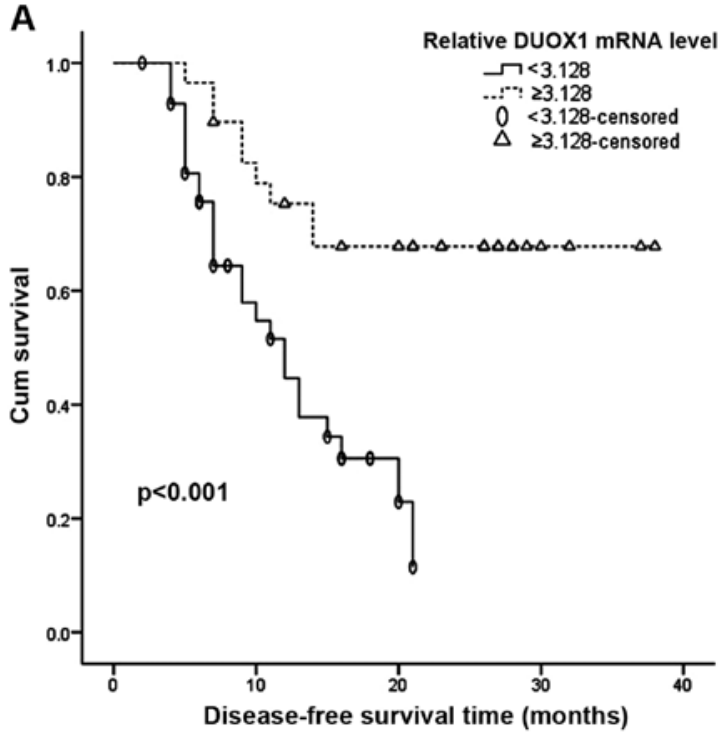

C

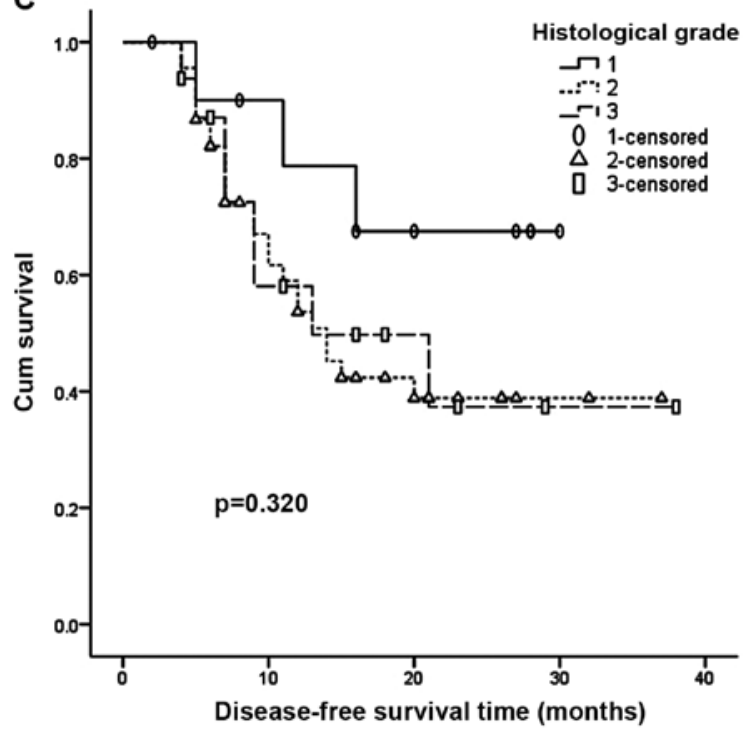

E

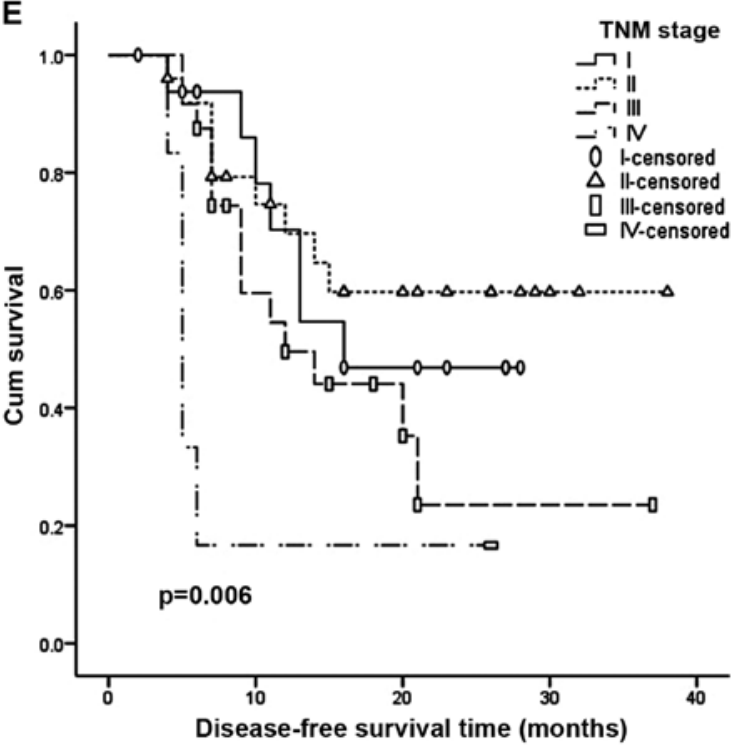

B

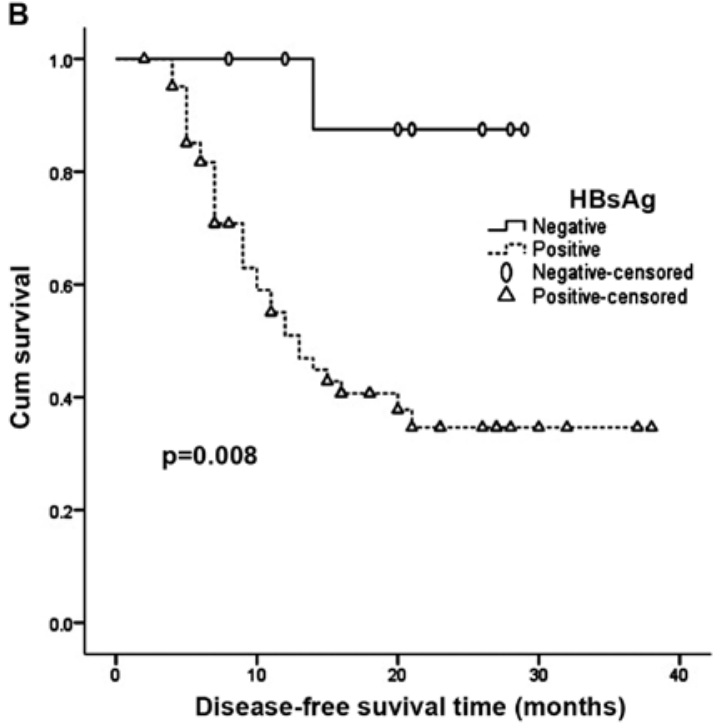

D

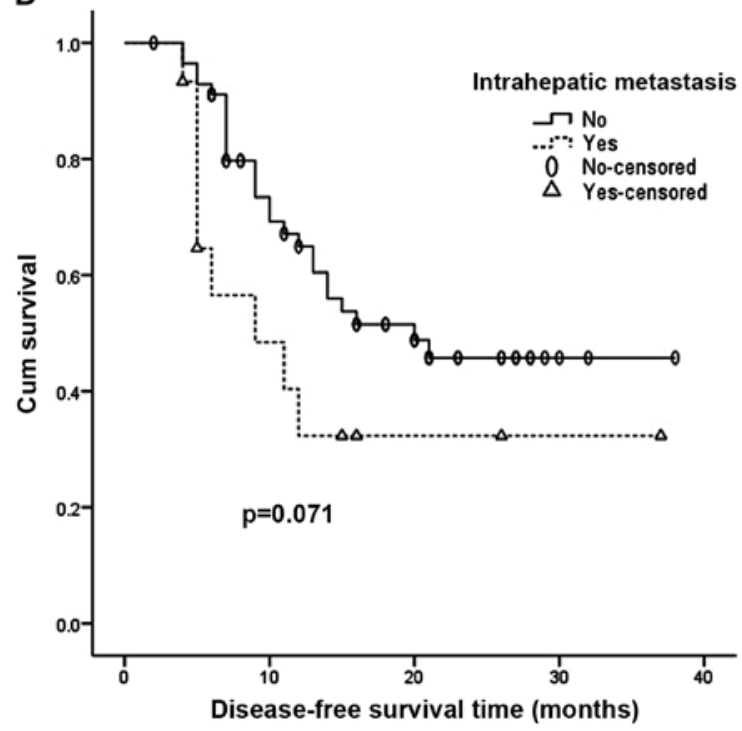

F

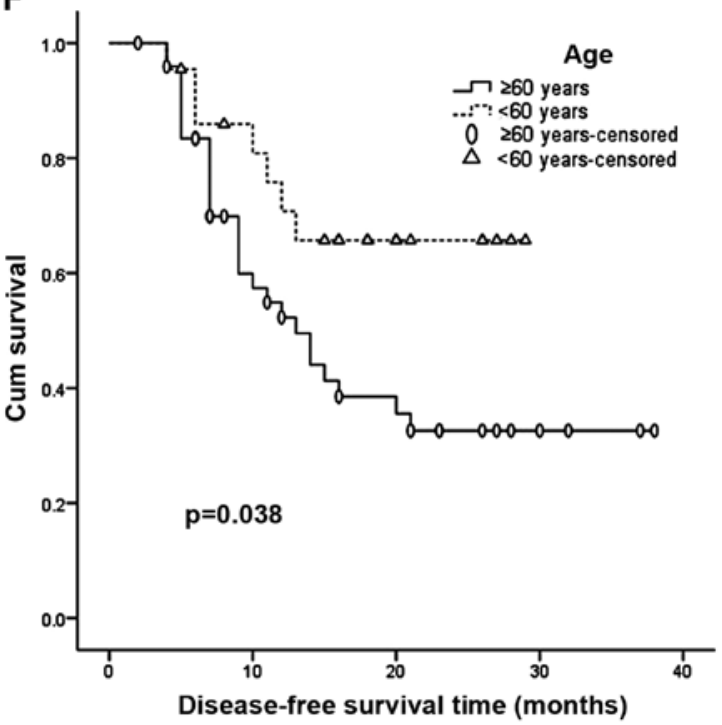

Figure 3. Impact of the clinicopathologic features on patient disease-free survival (DFS) after radical resection for HCC was evaluated using the Kaplan-Meier method (p-value according to the log-rank test). (A) Patients with high DUOX1 expression tended to have longer DFS compared with those with low DUOX1 expression. (B) HBsAg expression, (E) TNM stage and (F) age were significantly correlated with DFS. (C) Histological grade and (D) intrahepatic metastasis were not correlated with DFS 
A

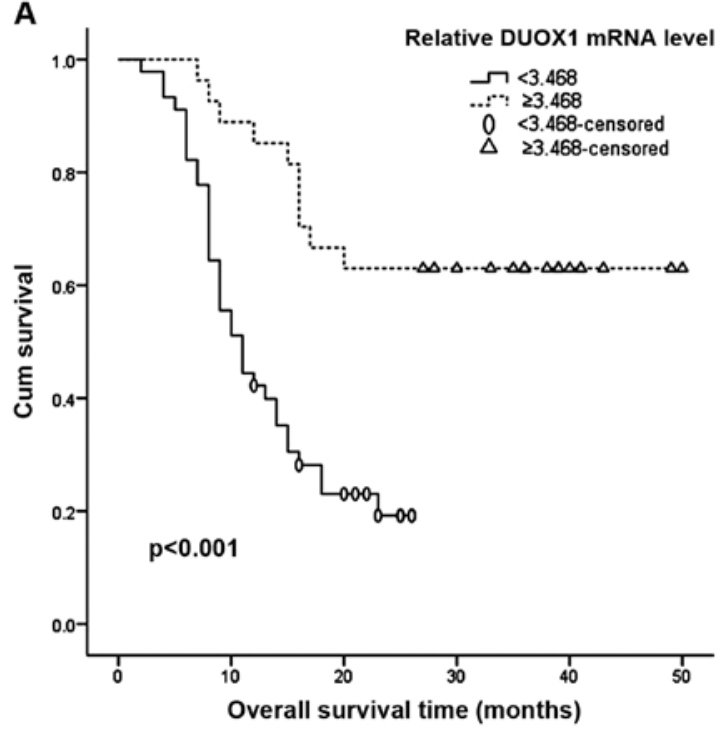

C
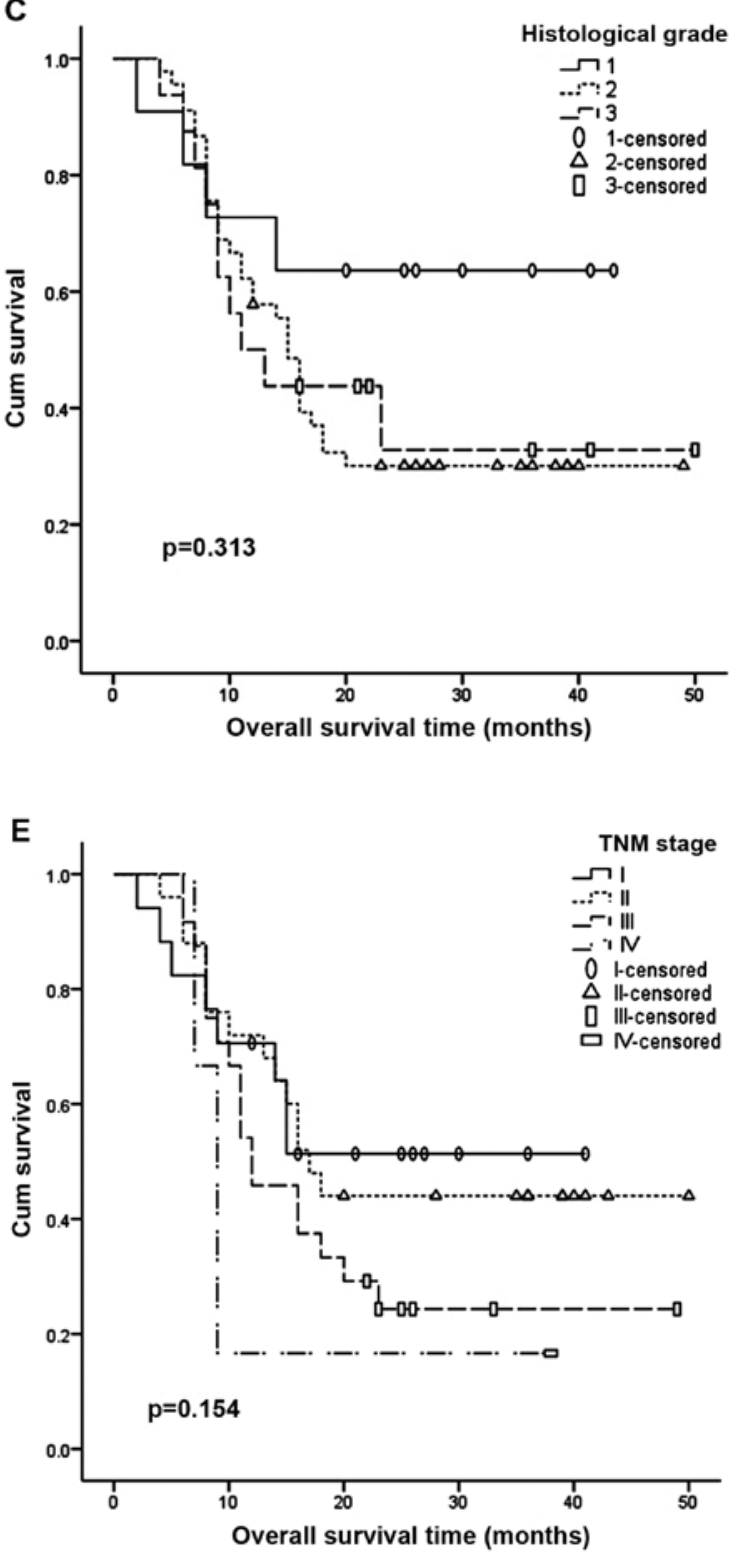

B

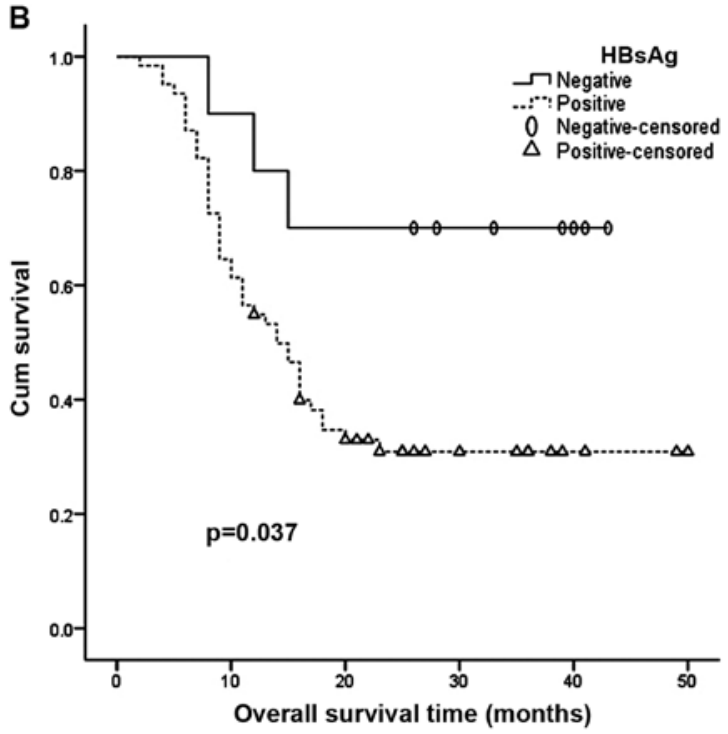

D

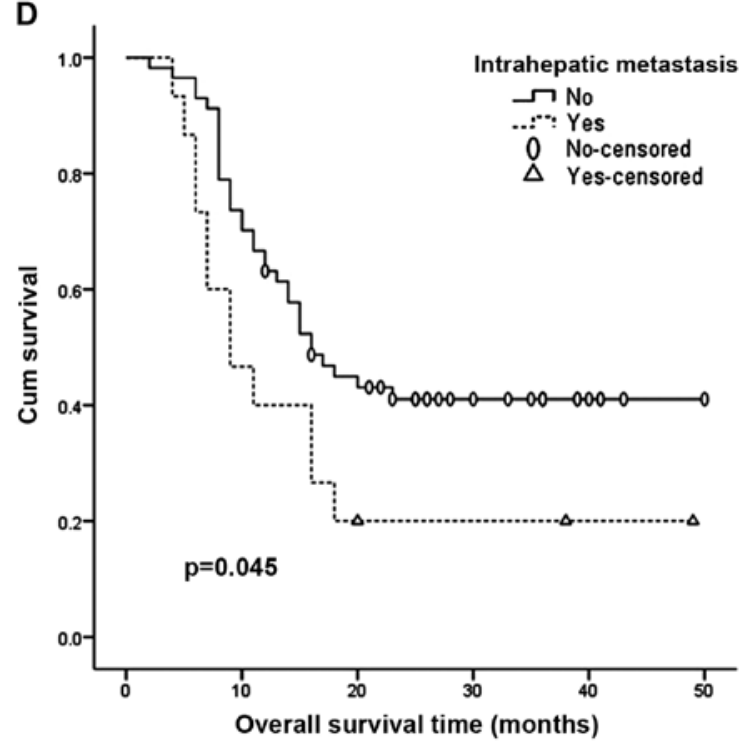

$\mathbf{F}$

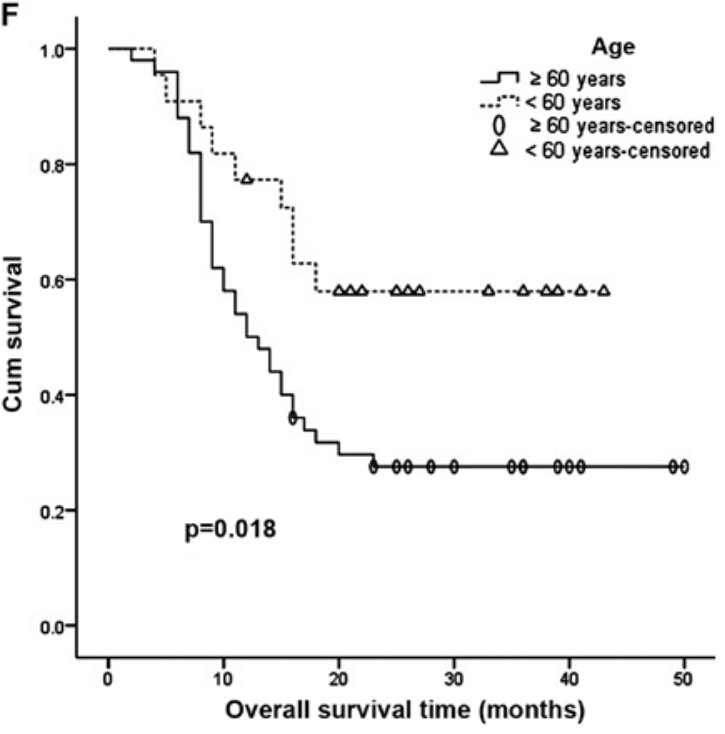

Figure 4. Impact of the clinicopathologic features on patient overall survival (OAS) after radical resection for HCC was evaluated using the Kaplan-Meier method (p-value according to the log-rank test). (A) Patients with high DUOX1 expression tended to have longer OAS compared with those with low DUOX1 expression. (B) HBsAg expression, (D) intrahepatic metastasis and (F) age were significantly correlated with OAS. (C) Histological grade and (E) TNM stage were not correlated with OAS. 
Table I. Univariate analysis of prognostic factors in patients with HCC as evaluated by disease-free survival and overall survival.

\begin{tabular}{|c|c|c|c|c|c|c|c|c|}
\hline \multirow[b]{2}{*}{ Variable } & \multirow{2}{*}{\multicolumn{2}{|c|}{ Number }} & \multicolumn{3}{|c|}{ Disease-free survival } & \multicolumn{3}{|c|}{ Overall survival } \\
\hline & & & $\mathrm{RR}(95 \% \mathrm{CI})$ & $\beta$ & P-value & $\mathrm{RR}(95 \% \mathrm{CI})$ & $\beta$ & P-value \\
\hline \multicolumn{9}{|l|}{ Gender } \\
\hline Male & 5 & 6 & $1.11(0.45-2.34)$ & 0.01 & 0.897 & $1.26(0.61-2.62)$ & 0.228 & 0.542 \\
\hline Female & 1 & 6 & Reference & & & Reference & & \\
\hline \multicolumn{9}{|l|}{ HBsAg } \\
\hline Positive & 6 & 2 & $8.82(1.21-64.64)$ & 2.177 & 0.032 & $3.17(0.98-10.24)$ & 1.153 & 0.054 \\
\hline Negative & 1 & 0 & Reference & & & Reference & & \\
\hline \multicolumn{9}{|l|}{ Age (years) } \\
\hline$<60$ & 5 & 0 & Reference & & & Reference & & \\
\hline$\geq 60$ & 2 & 2 & $2.30(1.01-5.29)$ & 0.834 & 0.049 & $2.31(1.11-4.80)$ & 0.836 & 0.025 \\
\hline \multicolumn{9}{|l|}{ Tumor size (cm) } \\
\hline$<5$ & 2 & 3 & Reference & & & Reference & & \\
\hline$\geq 5$ & 4 & 9 & $1.18(0.58-2.36)$ & 0.162 & 0.65 & $1.06(0.56-1.99)$ & 0.056 & 0.862 \\
\hline \multicolumn{9}{|c|}{ Intrahepatic metastasis } \\
\hline Yes & 1 & 5 & $1.96(0.91-4.20)$ & 0.673 & 0.084 & $1.92(0.99-3.72)$ & 0.651 & 0.054 \\
\hline No & 5 & 7 & Reference & & & Reference & & \\
\hline \multicolumn{9}{|c|}{ Histological grade } \\
\hline 1 or 2 & 5 & 6 & Reference & & & Reference & & \\
\hline 3 & 1 & 6 & $1.10(0.50-2.43)$ & 0.099 & 0.805 & $1.10(0.54-2.22)$ & 0.094 & 0.792 \\
\hline \multicolumn{9}{|l|}{ Tumor stage } \\
\hline I or II & 4 & 2 & Reference & & & Reference & & \\
\hline III or IV & 3 & 0 & $2.10(1.07-4.16)$ & 0.743 & 0.03 & $1.73(0.96-3.11)$ & 0.548 & 0.067 \\
\hline \multicolumn{9}{|c|}{ DUOX1 mRNA level } \\
\hline DFS OAS & DFS & OAS & & & & & & \\
\hline$<3.128<3.468$ & 43 & 45 & $3.65(1.67-7.96)$ & 1.294 & 0.001 & $3.69(1.81-7.54)$ & 1.307 & $<0.001$ \\
\hline$\geq 3.128 \geq 3.468$ & 29 & 27 & Reference & & & Reference & & \\
\hline
\end{tabular}

Table II. Multivariate analysis of prognostic factors in patients with HCC as evaluated by disease-free survival.

\begin{tabular}{|c|c|c|c|c|}
\hline Parameter & $\beta$ & $\mathrm{RR}$ & $95 \% \mathrm{CI}$ & P-value \\
\hline Relative DUOX1 mRNA level (<3.128 vs. $\geq 3.128)$ & 1.300 & 3.67 & $1.59-8.45$ & 0.002 \\
\hline Age ( $\geq 60$ vs. $<60$ years $)$ & 1.351 & 3.86 & $1.44-10.36$ & 0.007 \\
\hline Intrahepatic metastasis (yes vs. no) & 0.894 & 2.45 & $1.08-6.17$ & 0.046 \\
\hline TNM stage (III or IV vs. I or II) & 0.833 & 2.30 & $0.95-5.58$ & 0.065 \\
\hline HBsAg (positive vs. negative) & 1.287 & 3.62 & $0.43-30.21$ & 0.234 \\
\hline Histological grade (3 vs. 1 or 2 ) & 0.324 & 1.38 & $0.59-3.26$ & 0.459 \\
\hline Gender (male vs. female) & 0.397 & 1.49 & $0.62-3.55$ & 0.372 \\
\hline Tumor size ( $\geq 5 \mathrm{vs} .<5 \mathrm{~cm})$ & 0.246 & 1.28 & $0.53-3.11$ & 0.587 \\
\hline
\end{tabular}

RR, risk ratio; $95 \% \mathrm{CI}, 95 \%$ confidence interval; $\beta$, regression coefficient of the Cox proportional hazards model; P-value $<0.05$ according to univariate Cox proportional hazards model; histological grade, according to the three-tier grading scheme; TNM stage, Tumor-Node-Metastasis, according to the 7th edition of the AJCC (American Joint Committee on Cancer) Cancer Staging Manual. 
Table III. Multivariate analysis of prognostic factors in patients with HCC as evaluated by overall survival.

\begin{tabular}{llrr}
\hline Parameters & $\beta$ & RR & 95\% CI \\
\hline Relative DUOX1 mRNA level (<3.468 vs. $\geq 3.468)$ & 1.532 & 4.63 & $2.04-10.51$ \\
Age ( $\geq 60$ vs. $<60$ years) & 1.515 & 4.55 & $1.90-10.88$ \\
Intrahepatic metastasis (yes vs. no) & 1.132 & 3.10 & $1.30-7.40$ \\
TNM stage (III or IV vs. I or II) & 0.180 & 1.19 & $0.58-2.48$ \\
HBsAg (positive vs. negative) & 0.311 & 1.37 & $0.34-5.47$ \\
Histological grade (3 vs. 1 or 2) & 0.124 & 1.13 & $0.53-2.41$ \\
Gender (male vs. female) & 0.241 & 1.27 & $0.57-2.84$ \\
Tumor size ( $\geq 5$ vs. $<5$ cm) & 0.022 & 1.02 & $0.48-2.19$ \\
\hline
\end{tabular}

$\mathrm{RR}$, risk ratio; $95 \% \mathrm{CI}, 95 \%$ confidence interval; $\beta$, regression coefficient of the Cox proportional hazards model; P-value $<0.05$ according to univariate Cox proportional hazards model; histological grade, according to the three-tier grading scheme; TNM stage, Tumor-Node-Metastasis, according to the 7th edition of the AJCC (American Joint Committee on Cancer) Cancer Staging Manual.

Table IV. Components of the disease-free survival prediction score.

\begin{tabular}{lcc}
\hline Factors & $\begin{array}{c}\text { Score (rounded to } \\
\text { the nearest integer) }\end{array}$ Score origin \\
\hline
\end{tabular}

\section{DUOX1 mRNA level}

$$
\geq 3.128
$$$$
<3.128
$$

0

Age (years)

$<60$

$\geq 60$

2

Intrahepatic metastasis

\begin{tabular}{lll} 
No & 0 & \\
Yes & 1 & $0.894 / 0.894$ \\
\hline
\end{tabular}

Table V. Components of the overall survival prediction score.

\begin{tabular}{lcc}
\hline & $\begin{array}{c}\text { Score (rounded to } \\
\text { the nearest integer) }\end{array}$ & Score origin \\
\hline $\begin{array}{l}\text { DUOX10rs } \\
\geq 3.468\end{array}$ & 0 & \\
$\quad<3.468$ & 1 & $1.532 / 1.132$ \\
Age (years) & & \\
$\quad<60$ & 0 & $1.515 / 1.132$ \\
$\geq 60$ & 1 & \\
Intrahepatic metastasis & & $1.132 / 1.132$ \\
$\quad$ No & 0 & \\
Yes & 1 & \\
\hline
\end{tabular}
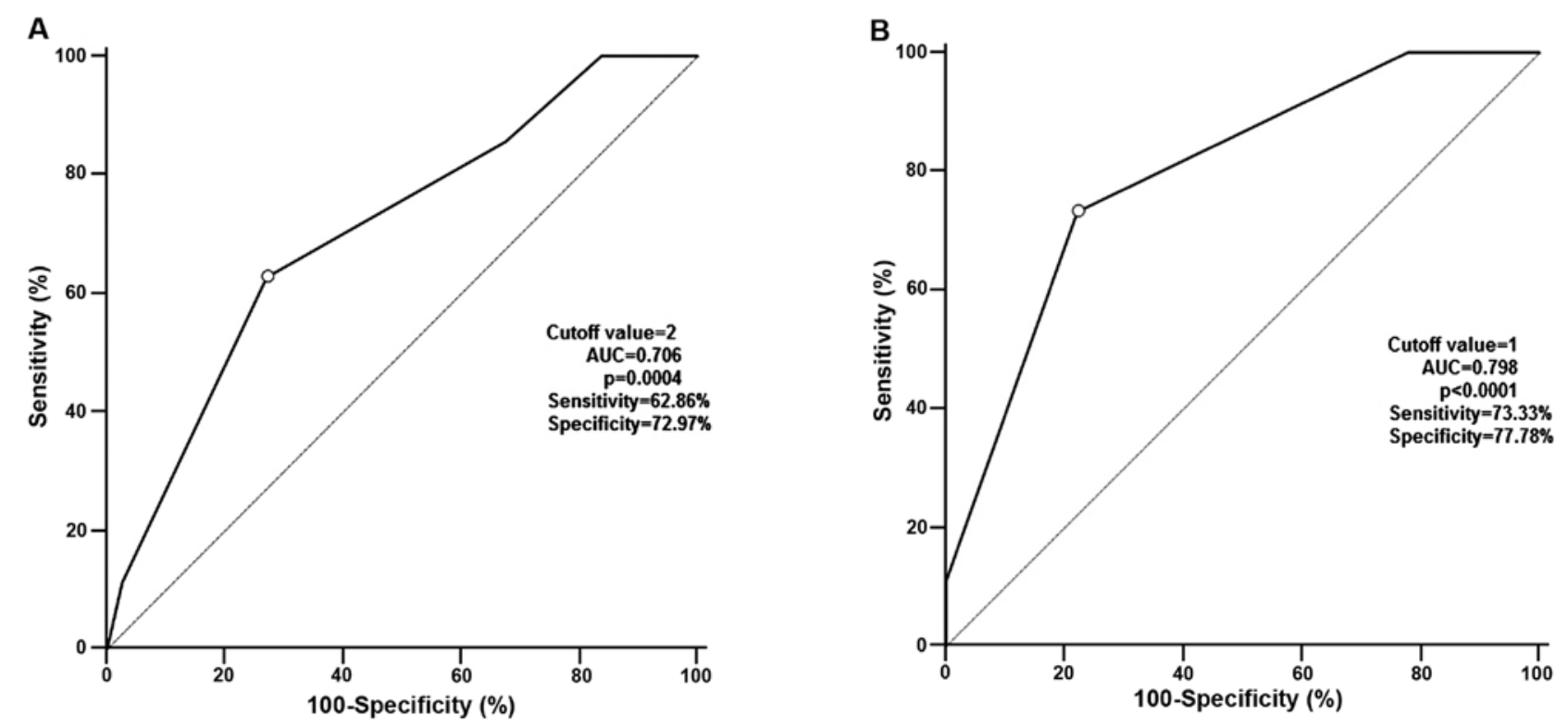

Figure 5. ROC curves with simplified risk score to predict the HCC patient prognosis. (A) ROC curve with simplified risk score to predict the recurrence of HCC in patients after surgery. (B) ROC curve with simplified risk score to predict the death of HCC patients after tumor resection. 
A

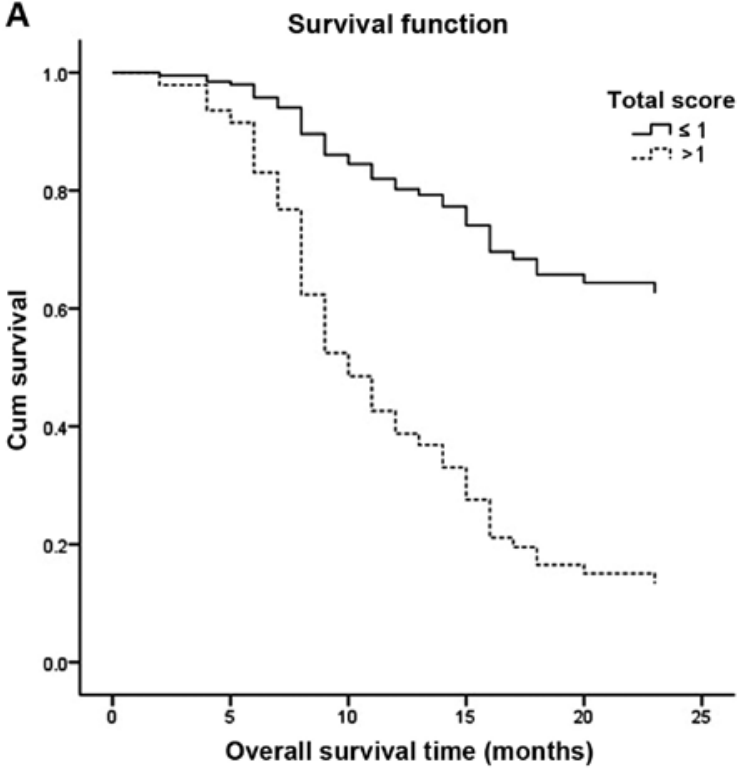

C

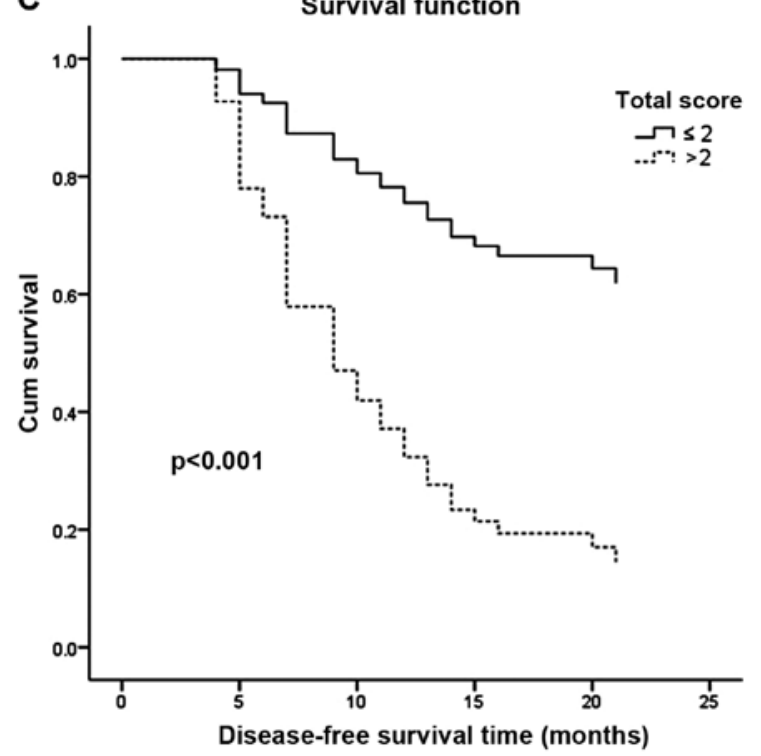

B

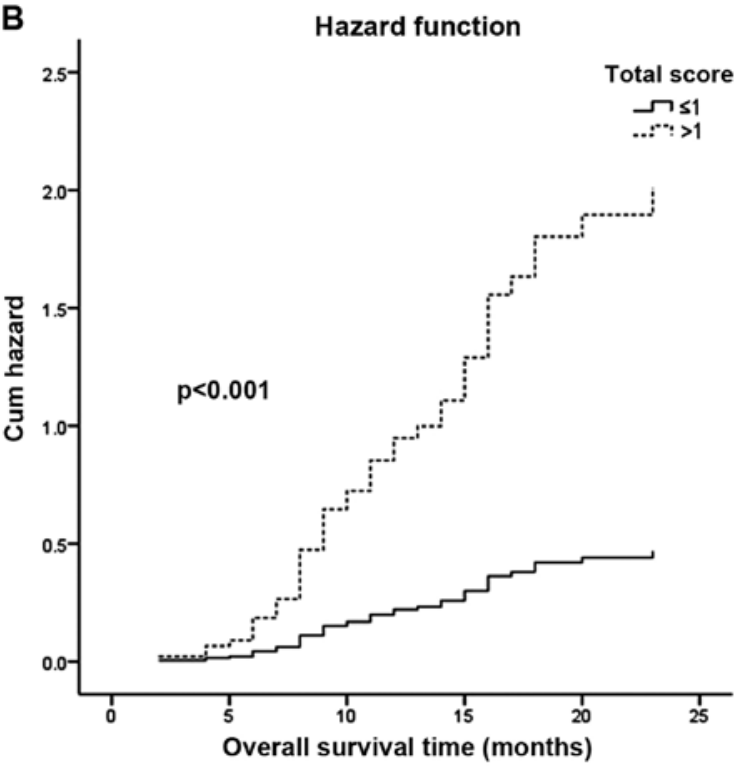

D

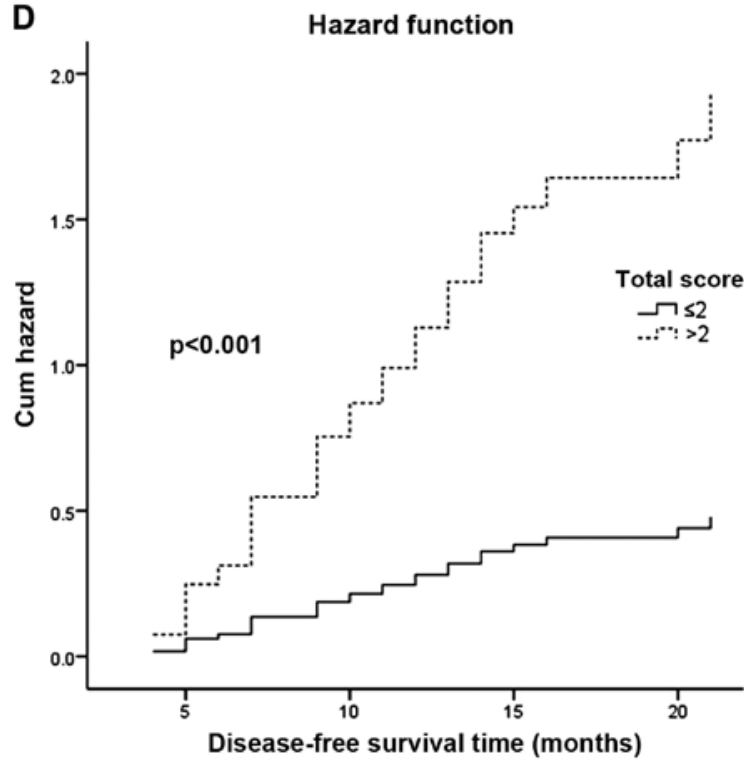

Figure 6. Iimpact of the total scoring system on disease-free survival and overall survival with Cox's regression analysis (p-value was confirmed with Cox proportional hazards model). Patients whose total score was $>2$ were more likely to relapse (C and D); patients with a total score $>1$ were apt to succumb to the disease (A and $\mathrm{B}$ ) than patients whose scores were $\leq 2$ and $\leq 1$.

stasis and one of the checkpoints, the G2/M checkpoint blocks the entry into mitosis when DNA is damaged (24). Based on our previous research, the liver cancer cell growth suppression induced by ectopic DUOX1 expression seemed to be caused by increasing $\mathrm{G} 2 / \mathrm{M}$ phase cell number (8), which implied that DUOX1 suppressed tumor cell growth through inducing G2/M phase cell cycle arrest. Furthermore, in a previous study, we verified that DUOX1 was frequently silenced by promoter hypermethylation in most liver cancer cell lines and primary HCC tissues, which suggest that epigenetic inactivation of DUOX1 is an important factor in the tumorigenesis of liver cancers (8). Regrettably, the associations between prognosis of liver cancer patients and DUOX1 expression were not identified in our previous research.
In the present study, we found that the DUOX1 mRNA expression was significantly decreased in the majority of primary HCCs that we examined compared with that in the non-tumor liver tissues (Fig. 1A). This result was consistent with our previous report. We further investigated the correlations between DUOX1 expression and clinicopathologic features of the liver cancer cases. DUOX1 expression was significantly correlated with $\mathrm{HBs} A g$ expression and hepatic cirrhosis (Fig. 1B and E). Notably, DUOX1 expression had no correlation with histological grade and tumor stage (Fig. 1C and D). Then two ROC curves of DUOX1 expression to predict DFS and OAS were performed. In terms of predicting DFS, the area under the ROC curve (AUC) was 0.653 for the DUOX1 mRNA level, and corresponding diag- 
nostic index including sensitivity was $77.14 \%$ and specificity was $54.05 \%$ (Fig. 2A). ROC analysis also identified the DUOX1 mRNA level as a predictive factor for OAS (AUC, 0.749; $\mathrm{p}=0.0001$; sensitivity, 77.78; specificity, 62.96\%) (Fig. 2B). Thus, we can conclude that DUOX1 is an efficient biomarker for HCC prognosis. We only measured the DUOX1 expression level in tissue and neglected expression of DUOX1 in the serum, which hindered the further study of the predictive efficacy of DUOX1 for HCC patient survival.

Further survival analysis with Kaplan-Meier method indicated that patients with high DUOX1 expression $(\geq 3.128)$ had a longer DFS compared to the patients with low expression of DUOX1 (<3.128) (Fig. 3A). Similarly, Fig. 4A shows that a high DUOX1 level $(\geq 3.468)$ was also correlated with longer OAS compared with the low DUOX1 level counterpart (<3.468). The HBsAg level and age were also significantly correlated with DFS (Fig. 3B and F) and OAS (Fig. 4B and F) according to the Kaplan-Meier analysis. Yet, intrahepatic metastasis was correlated with OAS only and TNM stage seemed merely related to DFS (Figs. 3D and E and 4D and E). More confusingly, univariate Cox regression analysis demonstrated that tumor stage and the HBsAg level all were merely correlated to DFS, and showed no association with OAS (Table I). Therefore, we speculated that interference may exist among these variables above. Fortunately, the influence of age and DUOX1 level on prognosis according to Table I corresponded to the study with Kaplan-Meier analysis.

In the present study, the univariate analysis with Kaplan-Meier method and Cox model was firstly chosen to detect important factors that may affect the prognosis of HCC patients, but the results according to these two methods had some contradictions. Thus, a multivariate analysis was needed to be performed to identify the authenticity and validity of the prognostic factors detected from the univariate analysis. Ultimately, we screened the prognostic factors, DUOX1 expression, age and intrahepatic metastasis, for DFS (Table II) and OAS (Table III). The data illustrated that the hazard ratios of DUOX1 expression for DFS and OAS were respectively 3.67 $(\mathrm{p}=0.002)$ and $4.63(\mathrm{p}<0.001)$, indicating that the group with lower DUOX1 expression may have an $\sim 3.67$-fold risk of liver cancer relapse and a 4.63 -fold risk of death. Nevertheless, the Cox regression analysis suggested that tumor stage, histological grade, gender and tumor size were not correlated with DFS and OAS, which is in conflict with other research (25). The reason leading to the difference between this research and other studies may be the small sample size. In our present study, only 72 HCC patients were included, which could hide the statistical significance of some variables in the Cox regression analysis.

In order to research the impact of DUOX1 in more detail expression, age and intrahepatic metastasis on DFS and OAS, we developed a simple score composed of three variables to predict the risk of HCC relapse and death after tumor resection. Patients with prediction score of $\leq 2 \mathrm{vs}$. $>2$ had a distinctly different risk of $\mathrm{HCC}$ relapse and with a total score of $\leq 1$ vs. $>1$ had a significantly different risk of HCC patient overall survival. Notably, patients with a total score $\leq 2$ had a low risk of HCC recurrence and with total score $\leq 1$ had a low risk for mortality (Fig. 6). Identification of patient risk for their prognosis could initiate an individualized surveillance program for HCC patients after tumor resection.
Tumor occurrence and development can be considered as the accumulation of gene mutations and epigenetic modifications. The predominant consequence of this accumulation is the activation of proto-oncogenes or silencing of tumor-suppressor genes (26). Consistent with previous reports that DUOX1 can inhibit the occurrence or development of malignant tumors through various mechanisms $(3,6)$, our results showed that the expression of DUOX1 in liver non-tumor tissue was significant higher than that in liver malignant tumor tissues. More importantly, we found that the patients with higher DUOX1 expression had better cumulative survival. These results together indicate that DUOX1 acts as a tumor suppressor in the development of hepatic carcinoma and could well be considered as a novel biomarker for prognosis and therapy in liver cancer. The scoring system including DUOX1 in this study can provide evidence to predict the recurrence and death of HCC patients after tumor resection.

In summary, this study generated valuable evidence that the high expression of DUOX1 in HCC leads to a better prognosis in terms of both DFS and OAS after radical resection. DUOX1 can be a useful predictor of survival in HCC patients. Moreover, the scoring system including DUOX1 acts as a predictive model first used in our study to predict HCC patient survival and this predictive model can be a potential prognostic tool for liver cancer patients.

\section{Acknowledgements}

We thank Dr Zhang Hao for providing the hepatoma samples from the 72 patients.

\section{References}

1. Yang JD and Roberts LR: Hepatocellular carcinoma: A global view. Nat Rev Gastroenterol Hepatol 7: 448-458, 2010.

2. Sakamoto M: Early HCC: Diagnosis and molecular markers. J Gastroenterol 44 (Suppl 19): 108-111, 2009.

3. Roy K, Wu Y, Meitzler JL, Juhasz A, Liu H, Jiang G, Lu J, Antony S and Doroshow JH: NADPH oxidases and cancer. Clin Sci (Lond) 128: 863-875, 2015.

4. De Deken X, Wang D, Many MC, Costagliola S, Libert F, Vassart G, Dumont JE and Miot F: Cloning of two human thyroid cDNAs encoding new members of the NADPH oxidase family. J Biol Chem 275: 23227-23233, 2000.

5. Fischer H: Mechanisms and function of DUOX in epithelia of the lung. Antioxid Redox Signal 11: 2453-2465, 2009.

6. Luxen S, Belinsky SA and Knaus UG: Silencing of DUOX NADPH oxidases by promoter hypermethylation in lung cancer. Cancer Res 68: 1037-1045, 2008.

7. Pulcrano M, Boukheris H, Talbot M, Caillou B, Dupuy C, Virion A, De Vathaire F and Schlumberger M: Poorly differentiated follicular thyroid carcinoma: Prognostic factors and relevance of histological classification. Thyroid 17: 639-646, 2007.

8. Ling Q, Shi W, Huang C, Zheng J, Cheng Q, Yu K, Chen S, Zhang $\mathrm{H}, \mathrm{Li} \mathrm{N}$ and Chen $\mathrm{M}$ : Epigenetic silencing of dual oxidase 1 by promoter hypermethylation in human hepatocellular carcinoma. Am J Cancer Res 4: 508-517, 2014.

9. Tong L, Chuang CC, Wu S and Zuo L: Reactive oxygen species in redox cancer therapy. Cancer Lett 367: 18-25, 2015.

10. Sablina AA, Budanov AV, Ilyinskaya GV, Agapova LS, Kravchenko JE and Chumakov PM: The antioxidant function of the p53 tumor suppressor. Nat Med 11: 1306-1313, 2005.

11. D'Autréaux B and Toledano MB: ROS as signalling molecules: Mechanisms that generate specificity in ROS homeostasis. Nat Rev Mol Cell Biol 8: 813-824, 2007.

12. Bhattacharyya $\mathrm{S}$ and Saha J: Tumour, oxidative stress and host $\mathrm{t}$ cell response: Cementing the dominance. Scand J Immunol 82: 477-488, 2015. 
13. Cabello CM, Bair WB III and Wondrak GT: Experimental therapeutics: Targeting the redox Achilles heel of cancer. Curr Opin Investig Drugs 8: 1022-1037, 2007.

14. Fruehauf JP and Meyskens FL Jr: Reactive oxygen species: A breath of life or death? Clin Cancer Res 13: 789-794, 2007.

15. Nagasue N, Kohno H, Chang YC, Taniura H, Yamanoi A Uchida M, Kimoto T, Takemoto Y, Nakamura T and Yukaya H: Liver resection for hepatocellular carcinoma. Results of 229 consecutive patients during 11 years. Ann Surg 217: 375-384, 1993.

16. Edge SB and Compton CC: The American Joint Committee on Cancer: The 7th edition of the AJCC cancer staging manual and the future of TNM. Ann Surg Oncol 17: 1471-1474, 2010.

17. Eisenhauer EA, Therasse P, Bogaerts J, Schwartz LH, Sargent D, Ford R, Dancey J, Arbuck S, Gwyther S, Mooney M, et al: New response evaluation criteria in solid tumours: Revised RECIST guideline (version 1.1). Eur J Cancer 45: 228-247, 2009.

18. Livak KJ and Schmittgen TD: Analysis of relative gene expression data using real-time quantitative PCR and the 2(-delta delta $C(T)$ ) method. Methods 25: 402-408, 2001.

19. Gokcan H, Savas N, Oztuna D, Moray G, Boyvat F and Haberal M: Predictors of survival in hepatocellular carcinoma patients. Ann Transplant 20: 596-603, 2015.
20. Hwang S, Lee YJ, Kim KH, Ahn CS, Moon DB, Ha TY, Song GW, Jung DH and Lee SG: The impact of tumor size on long-term survival outcomes after resection of solitary hepatocellular carcinoma: Single-institution experience with 2558 patients. J Gastrointest Surg 19: 1281-1290, 2015.

21. Nickel A, Kohlhaas M and Maack C: Mitochondrial reactive oxygen species production and elimination. J Mol Cell Cardiol 73: 26-33, 2014

22. Ramsey MR and Sharpless NE: ROS as a tumour suppressor? Nat Cell Biol 8: 1213-1215, 2006.

23. Berndtsson M,Hägg M, Panaretakis T, Havelka AM, Shoshan MC and Linder S: Acute apoptosis by cisplatin requires induction of reactive oxygen species but is not associated with damage to nuclear DNA. Int J Cancer 120: 175-180, 2007.

24. Taylor WR and Stark GR: Regulation of the G2/M transition by p53. Oncogene 20: 1803-1815, 2001.

25. Kao WY, Chao Y, Chang CC, Li CP, Su CW, Huo TI, Huang YH, Chang YJ, Lin HC and Wu JC: Prognosis of early-stage hepatocellular carcinoma: The clinical implications of substages of Barcelona Clinic Liver Cancer System based on a cohort of 1265 patients. Medicine (Baltimore) 94: e1929, 2015.

26. Hahn WC and Weinberg RA: Rules for making human tumor cells. N Engl J Med 347: 1593-1603, 2002. 